\title{
Proteomic analysis of chloroplasts from chlorophyll-deficient melon mutant
}

\author{
C.M. ZHAO ${ }^{* * *}$, J.Z. CUI**, X.G. LI*, B.J. CHEN ${ }^{* *}$, R.R. JIN**, and Z.Y. YU*,+ \\ Horticulture College, Northeast Agricultural University, No. 59 Mucai Street, Heilongjiang Province, \\ 150030 Harbin, China* \\ Harbin Academy of Agricultural Science, Harbin, China** \\ Life Science and Technology Institute, Harbin Normal University, Harbin, China***
}

\begin{abstract}
We aimed to understand the molecular-level changes occurring in the photosynthetic metabolic network in mutant chloroplasts. We performed comparative liquid chromatography-mass spectrometry protein profiling of wild-type (WT) and chlorophyll-deficient melon (Cucumis melo L.) mutants. We identified 390 differentially expressed proteins and 81 shared proteins varied significantly in abundance, of which 76 were upregulated and 5 were downregulated. Differentially expressed proteins were involved in the following biological processes: binding, catalytic, structural, transporter, and antioxidant activities. The mutant had 6.08-fold higher expression of glutamate-1-semialdehyde 2,1-aminomutase (GSAM), an enzyme that synthesizes the chlorophyll precursor, 5-aminolevulinic acid, and a 5.02-fold higher expression of pyridoxal biosynthesis protein, a GSAM coenzyme. An RNA recognition motif-containing protein (RRM) decreased in expression by 5.22-fold. This suggests that GSAM and RRM are particularly relevant to chlorophyll deficiency.
\end{abstract}

Additional key words: label-free protein quantification; photosynthetic pathways; proteome analysis; qRT-PCR.

\section{Introduction}

Chloroplasts perform various distinctive and essential functions for plant growth and development. The size of the plastid proteome ranges from 2,000 to 4,000 proteins in Arabidopsis thaliana L. and most of these proteins are nucleus-encoded (Jensen and Leister 2014). They are biosynthesized in the cytosol and then imported into chloroplasts. Chloroplasts have their own genomes, which encode approximately 100 proteins involved in photosynthesis, as well as the transcription and translation of the organelle's genes (Timmis et al. 2004). Relatively minor changes in a subset of chloroplasts would be sufficient to interrupt biological processes in the organelle. Chlorophyll (Chl) is an extremely important pigment that absorbs and converts light energy for plant use (Sandonà et al.1998). In Chldeficient mutants, a strong decrease or increase occurs in the abundance of the proteins involved in various metabolic pathways, including $\mathrm{Chl}$ biosynthesis and degradation metabolism, nitrogen metabolism, chloroplast development, and carbon and energy metabolism (Rutschow et al. 2008, Liu et al. 2016, Yu et al. 2016). For example, in the Arabidopsis ffc mutant (pale green), the levels of lightharvesting complex I (LHCI) and light-harvesting com- plex II (LHCII) antennae proteins and stromal chaperones (Cpn60, Cpn21, and Hsp70) increased, while the levels of chloroplast-encoded PSII core complex proteins (D1, D2, CP43, CP47, and cytochrome $b_{559 \alpha}$ ) and nucleus-encoded proteins (OEC16, OEC23, and OEC33) decreased (Rutschow et al. 2008). In particular, various RNAbinding proteins were upregulated in $f f c$ (Rutschow et al. 2008). In chloroplasts, initial transcripts undergo a multistep process mediated by ribonucleases directed by RNA-binding proteins (Stoppel and Meurer 2013). Thus, these proteins are crucial for the stability of chloroplast mRNA. This suggested that RNA-binding proteins are the mediators of chloroplast RNA metabolism, connecting transcription and translation in chloroplasts (Rutschow et al. 2008). In addition, members of the defense system that protect against reactive oxygen species (ROS), such as ascorbate peroxidase (APX) and superoxide dismutase (SOD), were detected at higher concentrations in Chldeficient chloroplasts. APX and SOD are the key enzymes catalyzing the conversion of $\mathrm{H}_{2} \mathrm{O}_{2}$ to $\mathrm{H}_{2} \mathrm{O}$. This indicates that the mutant is more sensitive to photoinduced damage and ROS accumulation (Liu et al. 2016).

Chl deficiency can affect chloroplast development, gene expression, protein synthesis, and signal transduction

\footnotetext{
Received 25 May 2018, accepted 28 November 2018.

${ }^{+}$Corresponding author; fax: +86045155190431, e-mail: yzy@neau.edu.cn

Abbreviations: ALA - 5-aminolevulinic acid; CA - carbonic anhydrase; CAT - catalase; Chl - chlorophyll; GluTR - glutamyl-tRNA reductase; GSAM - glutamate-1-semialdehyde 2,1-aminomutase; LOX - lipoxygenase; PEPC - phosphoenolpyruvate carboxylase; PPI - protein-protein interaction; RCA - Rubisco activase; ROS - reactive oxygen species; RRM - RNA recognition motif-containing protein; SA - salicylic acid; WT - wild-type.

Acknowledgements: This study was supported by an open project funding from Heilongjiang Provincial Key University Laboratory of Cold Area Vegetable Biology (Northeast Agricultural University).
} 
between the chloroplast and the nucleus (Bollivar et al. 2006, Carvalho et al. 2011, Kindgren et al. 2012, Motohashi et al. 2012, Kmiecik et al. 2016). Chl loss can occur from mutations to key enzymes in its synthesis or in either photosystem. The loss is accompanied by altered fluorescence and a decline in photosynthetic capacity, due to negative effects on photosystem assembly and lightharvesting activity (Rutschow et al. 2008, Motohashi et al. 2012, Peremarti et al. 2014, Satou et al. 2014). For example, glutamyl-tRNA reductase (GluTR, encoded by $H E M A$ ) is a key enzyme involved in the synthesis of 5-aminolevulinic acid (ALA), a Chl precursor (Hedtke et al. 2007). GluTR was significantly reduced when Arabidopsis thaliana was transformed with antisense HEMA1, which is accompanied by a reduction in the protochlorophyllide and Chl content (Kumar and Söll 2000). Similarly, Chl $a$ oxygenase dysfunction causes a rapid decrease in Chl $b$ content and can even halt $\mathrm{Chl} b$ synthesis entirely (Espineda et al. 1999). Moreover, mutations on genes associated with general chloroplast development also influence $\mathrm{Chl}$ synthesis to varying degrees. For instance, multiple studies have shown that the Chl content decreases in response to the mutation of enzymes from signal-transduction pathways unrelated to photosystems or of nuclear-encoded plastid-targeted RNA polymerases (Sawers et al. 2002, Hricová et al. 2006, Tomiyama et al. 2014, Liu et al. 2015).

Given the numerous processes that can affect $\mathrm{Chl}$ production, it is not surprising that Chl-deficient mutants are very common and are widely identified in plants (Greene et al. 1988, Li et al. 2013, Wang et al. 2014, Brestic et al. 2015). These leaf-color mutants are frequently used as morphological markers to simplify crop breeding. They are also ideal for studying photosynthesis and chloroplast functions.

Proteomics is a powerful tool to study the protein networks and molecular regulatory mechanisms of plant development and response to biotic and abiotic stresses (Singh et al. 2013, Bansal et al. 2016). Proteomic studies based on two-dimensional gel electrophoresis have been applied to investigate the mechanisms underlying leaf color determination in Hosta and Ginkgo biloba (Liu et al. 2016, $\mathrm{Yu}$ et al. 2016). However, 2-D PAGE is time-consuming and not amenable to automation for high-throughput analyses. In view of these limitations, alternative technologies with a high degree of efficiency and accuracy have been developed. Label-free proteomic analysis coupled with liquid chromatography-quadrupole mass spectrometry (LC-MS/MS) provides a rapid proteomic analytical method for the quantification of expressed proteins and measure-ment of significant changes within a plant proteome in a single experiment (Mohayeji et al. 2014). However, only a few studies have used the labelfree technology for the quantification of plastid proteomes (Fesenko et al. 2016, Lundquist et al. 2017). To the best of our knowledge, no previous studies have examined the changes that occur in the chloroplast proteomes of melon mutants. In this study, we employed the label-free LCMS/MS method to obtain a global view of differentially expressed proteins in a Chl-deficient melon mutant. Our results are expected to clarify the various pathways related to Chl synthesis and enhance the current understanding of how Chl influences chloroplast function.

\section{Materials and methods}

Plant materials and isolation of chloroplasts: The Chldeficient mutant pygl is a spontaneous mutant of the melon (Cucumis melo L.) cultivar Baishami 1. Plants were grown in a greenhouse at the Harbin Academy of Agricultural Science, Harbin, China. Chloroplasts were isolated from 6-week-old plants as previously described (Cline1986), using an isolation buffer (50 mM HEPES-KOH [pH 8.0], $330 \mathrm{mM}$ sorbitol, 2 mM EDTA-Na 2 [pH 8.0], 5 mM ascorbic acid, $5 \mathrm{mM}$ cysteine; $0.05 \%$ bovine serum albumin [BSA]), followed by a wash buffer (50 mM HEPES-KOH [pH 8.0], $330 \mathrm{mM}$ sorbitol, $2 \mathrm{mM}$ EDTA-Na $\mathrm{N}_{2}$ [pH 8.0]). Intact chloroplasts were purified on a $40 / 80 \%$ Percoll gradient (van Wijk et al. 2007). Chloroplast suspension (10 $\mu \mathrm{L})$ was added to $1 \mathrm{~mL}$ of an $80 \%$ acetone solution, mixed well, and centrifuged for $2 \mathrm{~min}$ at $3,000 \times g$. The supernatant was measured at $652 \mathrm{~nm}$ (Persee General Instruments, Beijing, China). Chl concentration was calculated according to Li et al. (2000).

Protein extraction and digestion: Chloroplast pellets were suspended in an ice-cold lysis buffer (4\% SDS, $100 \mathrm{mM}$ DTT, $150 \mathrm{mM}$ Tris-HCl [pH 8.0]) and then disrupted using a high-pressure homogenizer (Fastprep-24®, MP Biomedical, Santa Ana, CA, USA). Samples were ultrasonicated and boiled for $30 \mathrm{~min}$. The removal of debris was carried out via centrifugation for $40 \mathrm{~min}$ at $14,000 \times \mathrm{g}$ and $25^{\circ} \mathrm{C}$. The supernatant was quantified with a $B C A$ Protein Assay Kit (Bio-Rad, Berkeley, California, USA) and stored at $-80^{\circ} \mathrm{C}$. Each sample had three biological replicates. Purified chloroplast proteins $(250 \mu \mathrm{g})$ were mixed with DTT to yield a final concentration of $100 \mathrm{mM}$.

Samples $(250 \mu \mathrm{g})$ and $200 \mu \mathrm{L}$ of UA buffer ( $8 \mathrm{M}$ urea, $150 \mathrm{mM}$ Tris-HCl, $\mathrm{pH} 8.0)$ were added to ultrafiltration centrifuge tubes and centrifuged for $15 \mathrm{~min}$ at $14,000 \times \mathrm{g}$. The supernatant was discarded, and $100 \mu \mathrm{L}$ of IAA (50 mM IAA in UA) was added to the precipitate. This mixture was then shaken for $1 \mathrm{~min}$ at $600 \mathrm{rpm}$, incubated in darkness for $20 \mathrm{~min}$, and centrifuged again for $10 \mathrm{~min}$ at $14,000 \times g$. Next, another $100 \mu \mathrm{L}$ of UA buffer was added before samples were centrifuged for $10 \mathrm{~min}$ at $14,000 \times \mathrm{g}$; these two steps were repeated twice. After the addition of $100 \mu \mathrm{L}$ of $25 \mathrm{mM} \mathrm{NH}_{4} \mathrm{HCO}_{3}$, samples were centrifuged for $10 \mathrm{~min}$ at $14,000 \times \mathrm{g}$ twice. Finally, $40 \mathrm{~mL}$ of trypsin buffer $\left(2 \mu \mathrm{L}\right.$ trypsin in $\left.25 \mathrm{mM} \mathrm{NH} \mathrm{NCO}_{3}\right)$ was added, and samples were shaken for $1 \mathrm{~min}$ at $600 \mathrm{rpm}$ at $37^{\circ} \mathrm{C}$. The resulting peptides were collected as a filtrate, and the peptide content was estimated by UV light spectral density at $280 \mathrm{~nm}$.

LC-MS/MS analysis: Five micrograms of peptide was analyzed with LC-MS/MS. Peptides were separated in the nanoflow liquid system EASY-nLC1000 (Thermo Fisher Scientific, Grand Island, NY, USA). The EASY column SC200 $(150 \mu \mathrm{m} \times 100 \mathrm{~mm}, R P-C 18$, Thermo 
Fisher Scientific) was equilibrated using 100\% buffer A ( $2 \%$ acetonitrile and $0.1 \%$ formic acid). Samples were injected into EASY column SC001 traps $(150 \mu \mathrm{m} \times$ $20 \mathrm{~mm}$, RP-C18; Thermo Fisher Scientific) using a Surveyor autosampler (Thermo Fisher Scientific). Column chromatography was performed at a flow rate of $400 \mathrm{~nL} \mathrm{~min}^{-1}$. Samples were then eluted with a linear gradient: $0-100 \mathrm{~min}, 0-45 \%$ buffer B (84\% acetonitrile and $0.1 \%$ formic acid); 100-108 $\mathrm{min}, \quad 45-100 \%$ buffer B; and 108-120 min, 100\% buffer B. Next, for $120 \mathrm{~min}$, eluates were subjected to a $Q$-Exactive mass spectrometer (Thermo Finnigan) operating in positive ionization mode at a mass range of $300-1800 \mathrm{~m} / \mathrm{z}$. At $200 \mathrm{~m} / \mathrm{z}$, the MS1 resolution was 70,000 and the MS2 resolution was 17,500 .

Protein identification and quantification: Mass spectrometry data were analyzed in MaxQuant version 1.3.0.5 against Cucurbitales entries (6,316 total) from the Uniprot database (downloaded October 20, 2014). The search parameters were as follows: precursor mass window, $6 \mathrm{ppm}$; fragment ion mass tolerance, $20 \mathrm{ppm}$; and up to two missed cleavages allowed. Carbamidomethylation of cysteine was set as a fixed modification, while N-terminal protein acetylation and methionine oxidation were set as variable modifications. The false discovery rate cutoff for protein identification was 0.01 .

Bioinformatic analysis: Sequences of differentially expressed proteins were retrieved from the UniProtKB database (Release 2014_11) in FASTA format and searched against the SwissProt database (Cucurbitales) using the NCBI BLAST + client for Windows to find homologs (ratio $> \pm 4.0$ and $p<0.05$ ). The resulting normalized $\log _{2}$ ratio values were used for further analysis. Functional analysis of proteins was performed using Gene Ontology (GO) annotations and KEGG GENES (melons). The proteinprotein interaction network was evaluated using the String database against the $A$. thaliana database (https://stringdb.org/cgi/input.pl).

Quantitative reverse transcription PCR: Samples were ground to a fine powder in liquid nitrogen, and total RNA from samples was extracted using the RNAiso Plus Total RNA Isolation Kit (Takara, Kusatsu, Japan). Reverse transcription and real-time PCR were performed with the ReverTra Ace qPCR RT Kit (Toyobo, Japan). All reactions were run with three technical replicates and three biological replicates. RT-PCR was run in an $A B I-7500$ real-time system ( $A B I$, Foster City, California, USA). Relative gene expression was calculated using the $\Delta \Delta \mathrm{Ct}$ algorithm. The endogenous reference gene was $18 \mathrm{~S}$ RNA (Jain et al. 2006), and Y-1 was set as a reference sample for data normalization.

\section{Results}

Plant growth and chlorophyll content: Our objective was to investigate the protein expression pattern in chloroplasts from Chl-deficient leaves of the mutant melon.
Mutant leaves were yellow-green throughout growth and development, unlike the darker green of the WT. Plant height and petiole length were significantly lower in the mutant, manifesting as a dwarf phenotype and small leaves. Furthermore, the mutant exhibited slower growth. The Chl $a$ and $\mathrm{Chl} b$ concentrations were significantly higher in the WT than that in pygl, but the $\mathrm{Chl} a / b$ ratio was lower in the WT than that in pyg1 (Shao et al. 2013, Table 1). To obtain sufficient chloroplasts for proteome analysis, 6-week-old leaves from the mutant were used. The isolated intact chloroplast content of pyg 1 from $30 \mathrm{~g}$ of leaves was approximately one-third that of the WT.

Proteome analysis: To identify the protein expression pattern, chloroplast proteins were extracted from pyg 1 and WT plants in three independent biological experiments. The protein bands were stained using Coomassie Blue after SDS-PAGE electrophoresis for measuring the quality of the samples. Then the proteins were trypsinized and assessed by LC-MS/MS analysis in triplicate.

Six LC-MS/MS raw data files were imported in MaxQuant software (version 1.3.0.5) for a database search. Then, 2,569 unique peptides were identified to being derived from 390 proteins in six melon samples. Of these, 333 proteins were detected in both pyg1 and WT, with 81 shared proteins varying significantly in abundance $\left(\log _{2}(\mathrm{Y} / \mathrm{G}) \geq \pm 2.0, p<0.05 ; \mathrm{Y}-p y g 1, \mathrm{G}-\mathrm{WT}\right)$. Under these thresholds, 5 proteins were downregulated and 76 were upregulated in pygl (Table $1 \mathrm{~S}$, supplement). The molecular functions of the proteins in WT and pygl were classified by GO analysis. The results of GO analysis, enriched for the terms 'biological process', 'molecular function', and 'cellular component' are shown in Fig. 1. The proteins enriched in biological processes are involved in metabolic processes, cellular processes, singleorganism processes, cellular component organization or biogenesis, and response to stimulus, respectively. The proteins enriched in molecular function terms are involved in binding-related proteins, catalytic reactions, structural molecular activity, transporter activity, antioxidant activity, and electron carrier activity, respectively. The proteins enriched in cellular component terms include part of the cell, organelle, macromolecular complex, and membrane. Among the downregulated proteins, two are related to binding, one is the PSI reaction center subunit II, one is lipoxygenase (under 'metabolic process'), and the last is an extensin-like protein. Among the upregulated proteins, 63 proteins were involved in metabolic, 58 in cellular, and 45 in single-organism processes, respectively. Fifty-one proteins were only detected in the

Table 1. Pigment content (chlorophyll $a$ and $b$ ) of the pygl mutant and WT. Different letters within the same column indicate significant difference $(P<0.05)$ according to the $t$-test.

\begin{tabular}{llll}
\hline & $\begin{array}{l}\text { Chlorophyll } a \\
{\left[\mathrm{mg} \mathrm{g}^{-1}(\mathrm{FM})\right]}\end{array}$ & $\begin{array}{l}\text { Chlorophyll } b \\
{\left[\mathrm{mg} \mathrm{g}^{-1}(\mathrm{FM})\right]}\end{array}$ & Chl $a / b$ \\
\hline pyg1 & $0.60 \pm 0.02^{\mathrm{a}}$ & $0.22 \pm 0.01^{\mathrm{a}}$ & $2.7^{\mathrm{a}}$ \\
WT & $1.71 \pm 0.03^{\mathrm{b}}$ & $0.47 \pm 0.02^{\mathrm{b}}$ & $3.6^{\mathrm{b}}$ \\
\hline
\end{tabular}




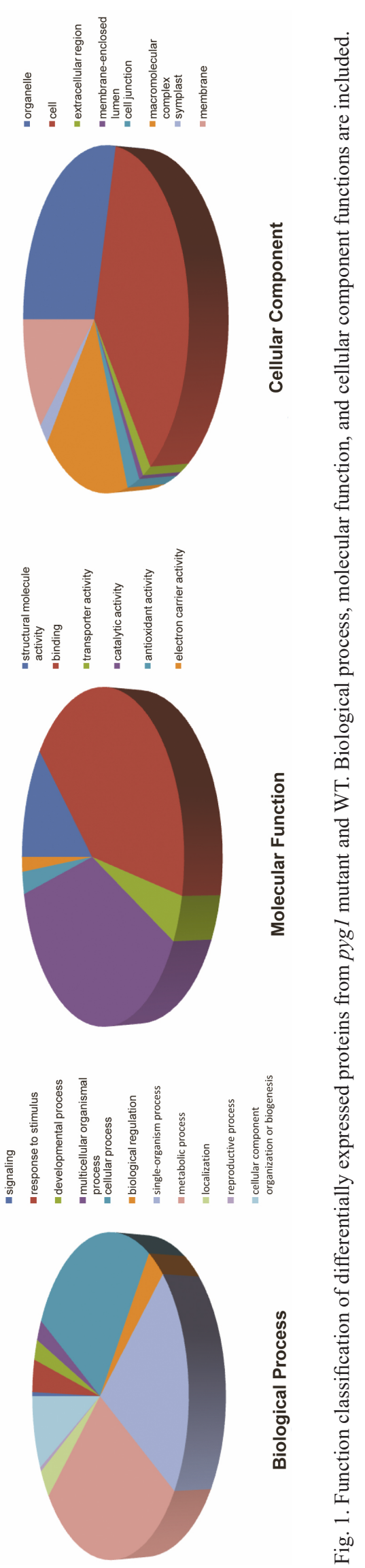

mutant, and 17 proteins were functionally limited; these function as a part of the membrane, which is an integral component of membrane, the transmembrane, in raffinose metabolism, galactose metabolism, glycoside hydrolase, and transporter activity. Proteins detected only in the WT include the GTP-binding protein, zeaxanthin epoxidase, p-coumaroyl-shikimate 3'-hydroxylase, NADH-ubiquinone oxidoreductase chain 3, phloem filament protein, and the $\mathrm{NAD}(\mathrm{P}) \mathrm{H}$-quinone oxidoreductase subunit $4 \mathrm{~L}$.

To further comprehend the enriched pathways of those differentially expressed proteins, $K E G G$ pathway analysis was performed. KAAS (KEGG Automatic Annotation Server) was adopted to compare the target proteins in the $K E G G$ GENES database (https://www.genome.jp/kegg/ genes.html). The results of the $K E G G$ analysis indicate that differentially expressed proteins were associated with $72 K E G G$ pathways, of which 9 were related to chloroplast function including carbon metabolism, ribosome, glyoxylate and dicarboxylate metabolism, carbon fixation, porphyrin and Chl synthesis, photosynthesis, etc. (Table 2S, supplement).

qRT-PCR analysis: Plant gene expression was regulated at the transcriptional and post-translational levels. Most of the proteins in pygl melon mutant were differentially expressed, including GSAM, photosynthetic system subunit proteins, and carbon-fixation-related proteins. In particular, the house-keeping protein, actin, was also significantly differentially expressed. In order to understand the changes in the protein levels, the transcript levels of nine genes with the highest differential expression (actin, $R R M$, $G S A M, C A T, R C A, p s a D$, pyridoxal biosynthesis protein, aminotransferase 1 , and alcohol dehydrogenase) were examined by qRT-PCR analysis. Actin and RRM did not differ at the RNA level between pygl and WT. RRM was downregulated at the protein level but not at the mRNA level. RCA exhibited the same trend as RRM in terms of mRNA- and protein-level expression. The remaining six genes had decreased mRNA expression in the mutant, while the protein expression levels increased (Fig. 1S).

Protein-protein interaction analysis: To further understand the functional associations of the identified proteins, a search for 37 differentially expressed proteins obtained from the experiment was carried out in the String database (https://string-db.org) against the Arabidopsis proteins (Fig. 2). There is a complex relationship between MDH, ICDH, THM1, GAPC2, PGK, CAT, HSP70, GPX2 proteins and other proteins. Several of these proteins are involved in carbon metabolism, glyoxylate and dicarboxylate metabolism, and carbon fixation pathways. LOX and $\mathrm{C} 4 \mathrm{H}$ are involved in the synthesis of oxylipin and 4-coumaric acid, which are the precursors of molecular signals for jasmonic acid and salicylic acid, respectively.

\section{Discussion}

In this study, proteomic and bioinformatic analyses of chloroplast proteins from Chl-deficient pygl mutant melon plants were performed. Analysis of chloroplast proteomic 


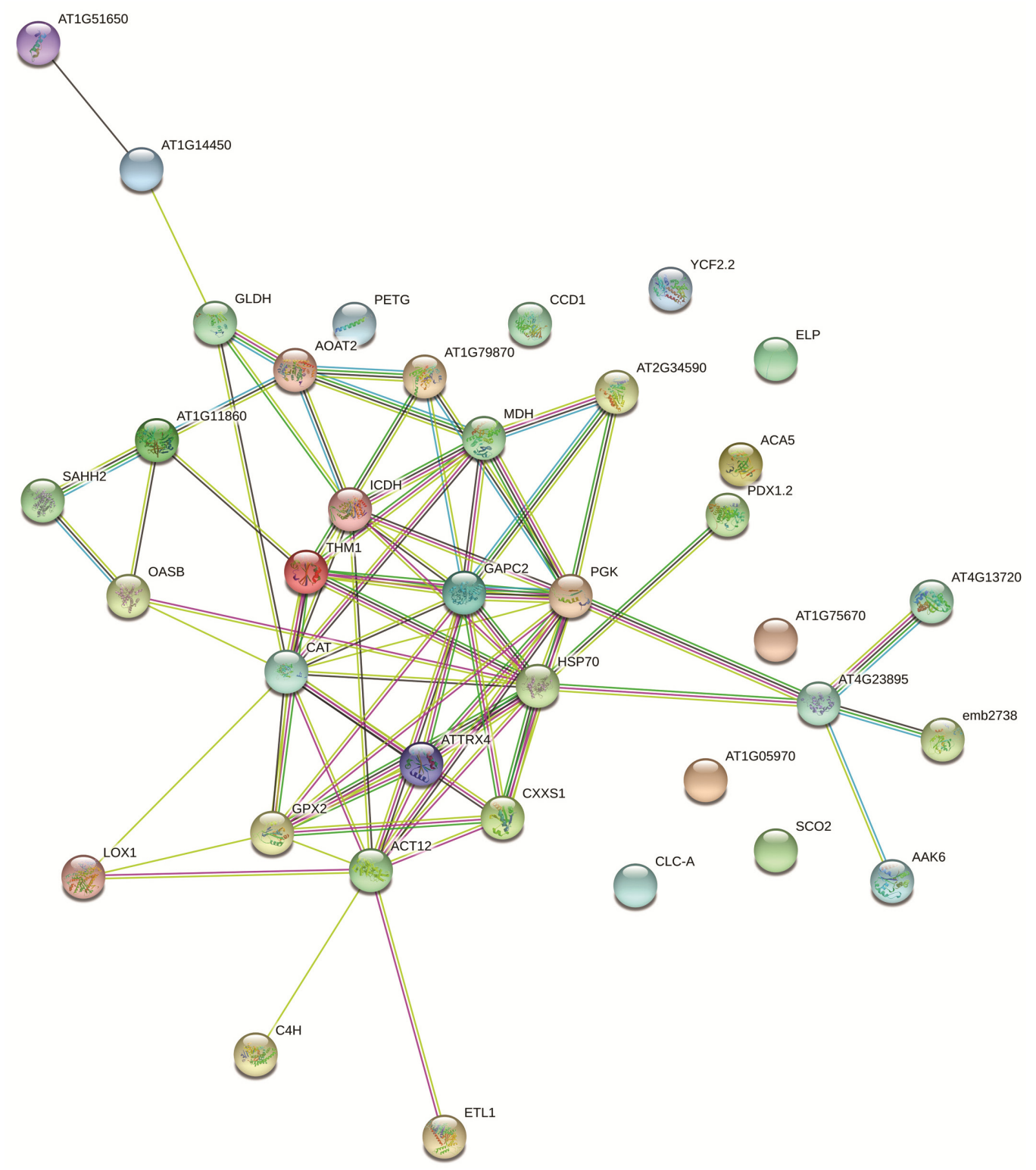

Fig. 2. Protein-protein interaction network of differentially expressed proteins analyzed by String 10. AAK6 - adenylatekinase; ACA5 - carbonic anhydrase; ACT12 - actin; AOAT2 - aminotransferase 1; AT1G05970 - putative RNA recognition motif-containing protein; AT1G11860 - aminomethyltransferase; AT1G14450 - NADH dehydrogenase; AT1G51650 - ATP synthase subunit a; AT1G75670 - DNA-directed RNA polymerase; AT1G79870 - glycerate dehydrogenase; AT2G34590 - chloroplast transketolase; AT4G13720 - pyrophosphatase; AT4G23895 - nucleoside diphosphate kinase; ATTRX4 - thioredoxin; C4H - cinnamate-4-hydroxylase; CAT - catalase; CCD1 - carotenoid cleavage dioxygenase; CLC-A - chloride channel a; CXXS1 - protein disulfide-isomerase; ELP - extensin-like protein; Emb2738 - GTP-binding protein; GAPC2 - glyceraldehyde 3-phosphate dehydrogenase; GLDH L-galactono-1,4-lactonedehydrogenase; GPX2 - glutathione peroxidase; HSP70 - chloroplast HSP70; ICDH -isocitrate dehydrogenase; $\mathrm{MDH}$ - malate dehydrogenase; OASB - cysteine synthase; PDX1.2 - pyridoxal biosynthesis protein; PETG - cytochrome $b_{6}-f$ complex iron-sulfur subunit; PGK - phosphoglycerate kinase; SAHH2 - adenosylhomocysteinase; THM1 - disulfide-isomerase; YCF2.2 protein Ycf2.

data revealed that the differentially expressed proteins are involved in multiple biological processes. The findings provide preliminary insights into the regulatory mechanisms of protein expression in melon mutants.

Most of proteins exhibited upregulated expression, but the transcription levels of specific genes analyzed showed the opposite expressional trend in the mutant. This finding suggests that the mutant negatively regulated the highly expressed proteins at transcription levels in order to control the content of the proteins in the chloroplasts. Actin is not highly expressed at the transcriptional level, which may be related to the characteristics of a house-keeping gene.

PPI analysis showed complex interactions between differentially expressed proteins. MDH, NADP-dependent 
isocitrate dehydrogenase, thioredoxin, GPX2, PGK, HSP70 are mainly involved in carbon fixation, photorespiration, and the glyoxylate cycle, transmembrane transport of proteins, reduction of the pentose-phosphate pathway, and the $\mathrm{C}_{4}$ pathway in chloroplasts, and play an important role in these processes.

Porphyrin and Chl biosynthesis pathway: We found glutamate-1-semialdehyde 2,1-aminomutase (GSAM) and a pyridoxal biosynthesis protein to be highly upregulated in the pygl mutant. Both enzymes are involved in the synthesis of porphyrins, the main component of Chl (Moser et al. 2001). GSAM catalyzes the final steps of ALA biosynthesis, and the inactivation or suppression of GSAM inhibits ALA biosynthesis in plants. The expression of antisense GSAM RNA has been found to cause a spectrum of Chl deficiencies in transgenic Brassica napus plants (Tsang et al. 2003).

Protoporphyrinogen IX oxidase also had upregulated expression. When the protoporphyrinogen IX oxidase activity was reduced or inhibited, the protoporphyrinogen IX accumulated in the chloroplasts or resulted in leakage into the cytoplasm. In the cytoplasm, protoporphyrinogen IX was oxidized to form singlet oxygen (Tripathy et al. 2007). The increase in protoporphyrinogen IX oxidase may increase the biosynthesis of $\mathrm{Chl}$ or may reduce the accumulation of protoporphyrinogen IX causing leakage into the cytoplasm.

Chl deficiency is caused by blockages in various steps of Chl synthesis. In the melon mutant, we expected key synthesis enzymes to exhibit reduced activity or content. However, contrary to our expectation, GSAM and protoporphyrinogen IX oxidase were upregulated at the protein level, and GSAM was the most abundant differentially expressed protein. This outcome suggests that $\mathrm{Chl}$ deficiency is primarily the result of changes to Chl-precursor synthesis, specifically the step that converts glutamate-1-semialdehyde to ALA.

Photosystems: The concentration of the PSI subunit protein PsaD was much higher in chloroplasts of the $p y g 1$ mutant. A contrasting trend was observed in the expression of other PSI subunit proteins (PsaA, PsaC, and PsaB). PsaA and PsaB subunits form a heterodimer that binds to the huge Chl molecule (Caffarri et al. 2014). The inhibited gene expression of $P s a A$ or $P s a B$ resulted in a reduction or deficiency of the PSI complex in the thylakoid. PsaD interacts directly with PsaC of PSI and helps PsaC properly assemble into PSI (Caffarri et al. 2014). The decrease in Chl molecular and subunit proteins might hamper the proper assembly of PSI while the increase in PsaD may enhance the stability of PSI.

The expression levels of Rieske $(2 \mathrm{Fe}-2 \mathrm{~S})$ protein (PetC) also increased. PetC is involved in electron transfer from PSII to PSI (Gubernator et al. 2006). Moreover, ferredoxin-NADP ${ }^{+}$reductase levels decreased slightly. This enzyme is involved in the final step of electron transfer from PSI to NADPH (Gubernator et al. 2006). This suggests that the defective PSI influenced electron transfer from PSII to PSI. The upregulated expression of PsaD,
Ycf2, and Ycf4 (PSI-assembly protein) may enhance the stability of the PSI complex and electron flow in the mutant, and electrons may be transmitted through other pathways, such as cyclic or pseudocyclic electron transfer.

Carbon fixation pathway: Three-carbon-sugar synthesis in the Calvin cycle occurs through the action of three main enzymes (Aubry et al. 2011), namely, Rubisco, phosphoglyceratekinase(PGK), andglyceraldehyde3-phosphate dehydrogenase (GAPDH) (Gontero et al. 1988), all of which exhibited significantly altered expression in the mutant. The activity of Rubisco is regulated by Rubisco activases (RCAs) (Erb and Zarzycki 2017). In absence of RCA, Rubisco activity cannot be properly maintained (Erb and Zarzycki 2017). Three classes of RCAs have been identified (Erb and Zarzycki 2017) and all of them were differentially expressed in the mutant showing different trends. One RCA was downregulated, while the other two were upregulated. This indicated different regulatory mechanisms of Rubisco activity by RCAs. These data suggest that the mutant exhibits improved photosynthetic carbon fixation capacity to synthesize glyceraldehyde3-phosphate, which is then used to form other carbohydrates. The expression of transketolase increased significantly. This indicates that the mutant continues to produce some Calvin cycle intermediates (i.e., ribulose-5-phosphate). These intermediate products in the Calvin cycle are precursors to nucleic acids, aromatic amino acids, and vitamin synthesis (Rochaix and Ramundo 2015).

In the mutant, there are three enzymes in the raffinose synthesis pathway that are differentially expressed, including alkaline alpha-galactosidase 3, alkaline alphagalactosidase, and raffinose synthase which were detected only in the mutant, but not in the WT. In plants, raffinose has a certain relationship with the plant stress tolerance. This may be one of the reasons why the pygl mutant has disease resistance.

Rubisco, phosphoenolpyruvate carboxylase (PEPC), malic enzyme (ME), and carbonic anhydrase (CA) were upregulated in melon mutant, which are important enzymes in the carbon fixation pathway of $\mathrm{C}_{4}$ plant. A significant increase of the four enzymes in the mutant indicates that there may be $\mathrm{C}_{4}$ pathway in melon cells.

Carbon metabolism: The differentially expressed proteins in the carbon metabolism pathway include ribulose-1,5diphosphate carboxylase large subunit, phosphoglycerate kinase, transketolase, glyceraldehyde-3-phosphate dehydrogenase (chloroplast), and aconitine hydratase, malate dehydrogenase, aconitate hydratase, cysteine synthase, isocitrate dehydrogenase (NADP), aminomethyl transferase, and phosphoenolpyruvate carboxylase. Carbon metabolism is closely related to carbon fixation, photorespiration, and amino acid synthesis. Malate dehydrogenase, isocitrate dehydrogenase, and phosphoenolpyruvate carboxylase all release $\mathrm{CO}_{2}$ during the catalytic process and thus provide a carbon source for carbon fixation. In addition to their role in providing $\mathrm{CO}_{2}$, these three enzymes may also be involved in the enhancement of photorespiration in the mutant, as the catalytic products 
of these enzymes are directly or indirectly involved in photorespiration. Increase in these enzymes may promote the photorespiration of cells, the main product of which is $\mathrm{H}_{2} \mathrm{O}_{2}$.

\section{Biosynthesis of amino acids and chloroplast ribosomal} proteins: Proteins are the basis of life activities and are involved in biological processes, such as synthesis and catabolism, material transport, and signal transmission. There are seven differentially expressed proteins involved in amino acid metabolism, cysteine synthase, aconitic acid hydratase, isocitrate dehydrogenase, phosphoglycerate kinase, and transketolase. Cysteine synthase is involved in the synthesis of cysteine, which is an important component of the antioxidant, glutathione peroxidase (GPX). GPX is also upregulated in the mutant, so it is speculated that its requirement for substrates would also increase. Aconitic acid hydratase and isocitrate dehydrogenase are involved in the synthesis of glutamic acid and synthesize arginine through glutamic acid. Glutamate is an amino acid closely related to the synthesis of $\mathrm{Chl}$ and is transformed into glutamyl-tRNA by glutamyl-tRNA synthetase (Chang et al. 1990). It is the substrate in the first step of Chl precursor ALA synthesis.

Most chloroplast ribosome subunit proteins were upregulated in the chloroplasts of the mutant. The considerable increase in protein translation in the mutant, suggests that ribosome quantity must also increase. Most components of translation in plastids (including $70 \mathrm{~S}$ ribosomes) have cyanobacterial homologs. Some studies have revealed a negative feedback system in the chloroplast. The cessation of chloroplast transcription or translation by inactivation of rps 12 leads to a marked increase in the abundance of the transcripts of most RNA polymerase and ribosomal protein genes (Rochaix and Ramundo 2015). In the mutant, a negative feedback system might exist, which may respond to Chl deficiency.

Catalase and lipoxygenase and cinnamate-4-hydroxylase: In the mutant, catalase and catalase isozyme 1 expression increased. These enzymes break down $\mathrm{H}_{2} \mathrm{O}_{2}$ into water and oxygen, constituting an important metabolic process for the reduction of oxidative stress. Under stress conditions, the lipids are peroxidated. LOX was one of the few proteins in the mutant whose expression decreased. Lipid peroxidation is initiated by LOX and is associated with the formation of hydroperoxy fatty acids, reactive carbonyl species (RCS), and ${ }^{1} \mathrm{O}_{2}$ (Jakhar et al. 2014). A highly upregulated protein is alkenal/one oxidoreductase (AOR), which catalyzes RCS using NADPH as the reductant in the PSII (Pospíšil and Yamamoto 2017), suggesting that the differentially expressed LOX and AOR in the mutant help decrease lipid peroxidation products in PSII. Furthermore, the levels of glutathione peroxidase and disulfide isomerase, enzymes with antioxidant properties, were also elevated in the mutant. These results suggest that $\mathrm{Chl}$ deficiency increased oxidative stress, possibly because of disruptions in photosynthesis pathways that elevated the normal production of ROS. Finally, Chl metabolism can also produce ROS. The Chl biosynthesis mutant $f l u$ accumulates protochlorophyllide, a compound that generates ${ }^{1} \mathrm{O}_{2}$ upon illumination (Kim and Apel 2013). These ROS are generally converted to $\mathrm{H}_{2} \mathrm{O}_{2}$, which is a versatile molecule that plays a dual role in cells. Under normal conditions, $\mathrm{H}_{2} \mathrm{O}_{2}$ is an important molecule involved in many biological processes. However, excess $\mathrm{H}_{2} \mathrm{O}_{2}$ is toxic to the plant. Thus, the synthesis of copious amounts of catalase might balance the $\mathrm{H}_{2} \mathrm{O}_{2}$ content in the mutant chloroplasts.

In addition to oxidizing fats, LOX is also involved in multiple metabolic pathways in plants. LOX is a key enzyme in the synthesis of jasmonic acid (Gasperini et al. 2015). Decreased expression of LOX reduces fat oxidation and may also reduce jasmonate synthesis. In the mutant, cinnamic acid 4-hydroxylase $(\mathrm{C} 4 \mathrm{H})$ catalyzing the hydroxylation of cinnamic acid to produce 4-coumaric acid was upregulated 2-fold, indicating that the mutant increased the synthesis of precursors of salicylic acid (SA, a signaling molecule, Chang et al. 1990). However, SA cannot transmit signals over long distances, and the longrange signal molecule $\mathrm{H}_{2} \mathrm{O}_{2}$ works with the SA to regulate signal transmission. The mutant may increase the synthesis of SA by inhibiting the synthesis of jasmonic acid and facilitating the $\mathrm{H}_{2} \mathrm{O}_{2}$ signal activating the expression of defense genes, thus increasing the resistance of the mutant.

RRM and actin: In the mutant, a significant decrease in an RNA recognition motif-containing (RRM) protein was found to have unclear functions. With two motifs, the protein is most similar to At5g46840 (another RRMcontaining protein with two motifs) (Sun et al. 2013). In maize, mutations in Zm-orrm 1, an rrm homolog, resulted in a paler green phenotype (Sun et al. 2013). Thus, it is possible that RRM downregulation is a key cause of Chl deficiency in pygl melon mutant.

Actin was significantly upregulated in the mutant. In chloroplasts, this protein is important for stromule formation (Natesan et al. 2005). The presence of stromules in chloroplasts has been found to be inversely correlated with the size and number of chloroplasts. Chl deficiency stunts development and reduces chloroplast number. The formation of stromules provides a much larger plastid surface area for exchanging metabolites or signaling molecules (Natesan et al. 2005). Chloroplast ROS production induces stromule formation, causing $\mathrm{H}_{2} \mathrm{O}_{2}$ to relocate from chloroplasts to the nucleus via stromules (Brunkard et al. 2015, Caplan et al. 2015). Therefore, the expression of nuclear genes and proteins transferred into chloroplasts might be related to the upregulation of actin expression in the mutant.

The actin depolymerization factor protein was detected in the mutant but was not detected in the WT. The binding of this protein to actin is the major regulator of actin patterns responding to oxidative stress, it also has the ability to resist both biological and abiotic stresses (Fu et al.2014, Henty-Ridilla et al. 2014). This may also be one of the reasons that the mutant showed a strong resistance to powdery mildew and exhibited drug resistance.

Conclusions: Chlorophyll deficiency in the melon altered 
both external morphology and physiological processes. Our exploration of the chloroplast proteome using label-free quantification revealed that the pygl mutant possesses many altered metabolic pathways. The most notable ones included those involved in porphyrin/chlorophyll biosynthesis, ribosomal protein synthesis, and carbon fixation. Although most proteins in these pathways were upregulated, their mRNA expression was downregulated. Several downregulated proteins (such as RRM) had functions that were unclear, and thus, their correlation to chlorophyll synthesis should be examined in future studies. However, we also observed a strong increase in proteins with known connections to chlorophyll production, such as GSAM. This protein is involved in ALA (chlorophyll precursor) synthesis, suggesting that this part of the pathway is particularly relevant to chlorophyll deficiency. The mutation caused photosystem damage and oxidative stress. Our study provides valuable data on the mechanisms of chlorophyll-related pathways and enhances the existing knowledge of chloroplast function.

\section{References}

Aubry S., Brown N.J., Hibberd J.M.: The role of proteins in $\mathrm{C}_{3}$ plants prior to their recruitment into the $\mathrm{C}_{4}$ pathway. - J. Exp. Bot. 62: 3049-3059, 2011.

Bansal M., Sharma M., Kanwar P., Goyal A.: Recent advances in proteomics of cereals.- Biotechnol. Genet. Eng. 32: 1-17, 2016.

Bollivar D.W.: Recent advances in chlorophyll biosynthesis. Photosynth. Res. 90: 173-194, 2006.

Brestic M., Zivcak M., Kunderlikova K. et al.: Low PSI content limits the photoprotection of PSI and PSII in early growth stages of chlorophyll $b$-deficient wheat mutant lines. Photosynth. Res. 125: 151-166, 2015.

Brunkard J.O., Runkel A.M., Zambryski P.C.: Chloroplasts extend stromules independently and in response to internal redox signals. - P. Natl. Acad. Sci. USA 112: 10044-10049, 2015.

Caffarri S., Tibiletti T., Jennings R.C., Santabarbara S.: A comparison between plant photosystem I and photosystem II architecture and functioning. - Curr. Protein Pept. Sc. 15: 296-331, 2014.

Caplan J.L., Kumar A.S., Park E. et al.: Chloroplast stromules function during innate immunity. - Dev. Cell. 34: 45-57, 2015.

Carvalho R.F., Takaki M., Azevedo R.A.: Plant pigments: the many faces of light perception. - Acta Physiol. Plant. 33: 241-248, 2011.

Chang T.E., Wegmann B., Wang W.Y.: Purification and characterization of glutamyl-tRNA synthetase: An enzyme involved in chlorophyll biosynthesis. - Plant Physiol. 93: 1641-1649, 1990.

Cline K.: Import of proteins into chloroplasts. Membrane integration of a thylakoid precursor protein reconstituted in chloroplast lysates. - J. Biol. Chem. 261: 14804-14810, 1986.

Erb T.J., Zarzycki J.: A short history of RubisCO: the rise and fall (?) of Nature's predominant $\mathrm{CO}_{2}$ fixing enzyme. - Curr. Opin. Biotech. 49:100-107, 2017.

Espineda C.E., Linford A.S., Devine D., Brusslan J.A.: The $A t C A O$ gene, encoding chlorophyll $a$ oxygenase, is required for chlorophyll $b$ synthesis in Arabidopsis thaliana. - P. Natl. Acad. Sci. USA 96: 10507-10511, 1999.

Fesenko I., Seredina A., Arapidi G. et al.: The Physcomitrella patens chloroplast proteome changes in response to proto- plastation. - Front. Plant Sci. 7: 1661, 2016.

Fu Y., Duan X., Tang C., Wang X. et al.: TaADF7, an actindepolymerizing factor, contributes to wheat resistance against Puccinia striiformis f. sp. tritici. - Plant J. 78: 16-30, 2014.

Gasperini D., Chauvin A., Acosta I.F. et al.: Axial and radial oxylipin transport. - Plant Physiol. 169: 2244-2254, 2015.

Gontero B., Cárdenas M.L., Ricard J.: A functional five-enzyme complex of chloroplasts involved in the Calvin cycle. - Eur. J. Biochem. 173: 437-443, 1988.

Greene B.A., Allred D.R., Morishige D.T., Staehelin L.A.: Hierarchical response of light harvesting chlorophyll-proteins in a light-sensitive chlorophyll $b$-deficient mutant of maize. Plant. Physiol. 87: 357-364, 1988.

Gubernator B., Króliczewski J., Kallas T., Szczepaniak A.: Ironsulfur cluster reconstitution of spinach chloroplast Rieske protein requires a partially prefolded apoprotein. - BBAProteins Proteom. 1764: 735-742, 2006.

Hedtke B., Alawady A., Chen S. et al.: HEMA RNAi silencing reveals a control mechanism of ALA biosynthesis on $\mathrm{Mg}$ chelatase and Fe chelatase. - Plant Mol. Biol. 64: 733-742, 2007.

Henty-Ridilla J.L., Li J., Day B., Staiger C.J.: ACTIN DEPOLYMERIZING FACTOR4 regulates actin dynamics during innate immune signaling in Arabidopsis. - Plant Cell 26: 340-352, 2014.

Hricová A., Quesada V., Micol J.L.: The SCABRA3 nuclear gene encodes the plastid RpoTp RNA polymerase, which is required for chloroplast biogenesis and mesophyll cell proliferation in Arabidopsis. - Plant Physiol. 141: 942-956, 2006.

Jain M., Nijhawan A., Tyagi A.K., Khurana J.P.: Validation of housekeeping genes as internal control for studying gene expression in rice by quantitative real-time PCR. - Biochem. Bioph. Res. Co. 345: 646-651, 2006.

Jakhar S., Mukherjee D.: Chloroplast pigments, proteins, lipid peroxidation and activities of antioxidative enzymes during maturation and senescence of leaves and reproductive organs of Cajanus cajan L. - Physiol. Mol. Biol. Pla. 20: 171-180, 2014.

Jensen P.E., Leister D.: Chloroplast evolution, structure and functions. - F1000Prime Rep. 6: 40, 2014.

Kim C., Apel K.: Singlet oxygen-mediated signaling in plants: moving from flu to wild type reveals an increasing complexity. - Photosynth. Res. 116: 455-464, 2013.

Kmiecik P., Leonardelli M., Teige M.: Novel connections in plant organellar signalling link different stress responses and signalling pathways. - J. Exp. Bot. 67: 3793-3807, 2016.

Kindgren P., Kremnev D., Blanco N.E. et al.: The plastid redox insensitive 2 mutant of Arabidopsis is impaired in PEP activity and high light-dependent plastid redox signalling to the nucleus. - Plant J. 70: 279-291, 2012.

Kumar A.M., Söll D.: Antisense HEMA1 RNA expression inhibits heme and chlorophyll biosynthesis in Arabidopsis. Plant Physiol. 122: 49-56, 2000.

Li N., Jia J., Xia C. et al.: Characterization and mapping of novel chlorophyll deficient mutant genes in durum wheat. Breeding Sci. 63: 169-175, 2013.

Li H.S., Sun Q., Zhao S.J. et al.: [The Experiment Principle and Technique on Plant Physiology and Biochemistry.] Pp. 134. Higher Education Press, Beijing 2000. [In Chinese]

Liu J., Zhou W., Liu G. et al.: The conserved endoribonuclease $\mathrm{YbeY}$ is required for chloroplast ribosomal RNA processing in Arabidopsis. - Plant Physiol. 168: 205-221, 2015.

Liu X., Yu W., Wang G. et al.: Comparative proteomic and physiological analysis reveals the variation mechanisms of leaf coloration and carbon fixation in a xantha mutant of Ginkgo biloba L. - Int. J. Mol. Sci. 17: 1794, 2016. 
Lundquist P.K., Mantegazza O., Stefanski A. et al.: Surveying the oligomeric state of Arabidopsis thaliana chloroplasts. Mol. Plant 10: 197-211, 2017.

Mohayeji M., Capriotti A.L., Cavaliere C. et al.: Heterosis profile of sunflower leaves: a label free proteomics approach. J. Proteomics 99: 101-110, 2014.

Moser J., Schubert W.D., Beier V. et al.: V-shaped structure of glutamyl-tRNA reductase, the first enzyme of tRNA-dependent tetrapyrrole biosynthesis. - EMBO J. 20: 6583-6590, 2001.

Motohashi R., Rödiger A., Agne B. et al.: Common and specific protein accumulation patterns in different albino/pale-green mutants reveals regulon organization at the proteome level. Plant Physiol. 160: 2189-2201, 2012.

Natesan S.K., Sullivan J.A., Gray J.C.: Stromules: a characteristic cell-specific feature of plastid morphology. - J. Exp. Bot. 56: 787-797, 2005

Peremarti A., Marè C., Aprile A. et al.: Transcriptomic and proteomic analyses of a pale-green durum wheat mutant shows variations in photosystem components and metabolic deficiencies under drought stress. - BMC Genomics 15: 125, 2014.

Pospíšil P., Yamamoto Y.: Damage to photosystem II by lipid peroxidation products. - BBA-Gen. Subjects 1861: 457-466, 2017.

Rochaix J.D., Ramundo S.: Conditional repression of essential chloroplast genes: Evidence for new plastid signaling pathways. - Biochim. Biophys. Acta 1847: 986-992, 2015.

Rutschow H., Ytterberg A.J., Friso G. et al.: Quantitative proteomics of a chloroplast SRP54 sorting mutant and its genetic interactions with CLPC1 in Arabidopsis. - Plant Physiol. 148: 156-175, 2008.

Sandonà D., Croce R., Pagano A. et al.: Higher plants light harvesting proteins. Structure and function as revealed by mutation analysis of either protein or chromophore moieties. BBA-Bioenergetics 1365: 207-214, 1998.

Satou M., Enoki H., Oikawa A. et al.: Integrated analysis of transcriptome and metabolome of Arabidopsis albino or pale green mutants with disrupted nuclear-encoded chloroplast proteins. - Plant Mol. Biol. 85: 411-428, 2014.
Sawers R.J.H., Linley P.J., Farmer P.R. et al.: Elongated mesocotyl1, a phytochrome-deficient mutant of maize. - Plant Physiol. 130: 155-163, 2002.

Shao Q., Yu Z.Y., Li X.G. et al.: [Studies on internal physiological and biochemical changes of xantha mutant in melon leaves.] China Vegetables 14: 59-65, 2013. [In Chinese]

Singh R., Jwa N.S.: Understanding the responses of rice to environmental stress using proteomics. - J. Proteome Res. 12: 4652-4669, 2013.

Stoppel R., Meurer J.: Complex RNA metabolism in the chloroplast: an update on the psbB operon. - Planta 237: 441-449, 2013.

Sun T., Germain A., Giloteaux L. et al.: An RNA recognition motif-containing protein is required for plastid RNA editing in Arabidopsis and maize. - P. Natl. Acad. Sci. USA 110: 1169-1178, 2013.

Timmis J.N., Ayliffe M.A., Huang C.Y., Martin W.: Endosymbiotic gene transfer: organelle genomes forge eukaryotic chromosomes. - Nat. Rev. Genet. 5: 123-135, 2004.

Tomiyama M., Inoue S., Tsuzuki T. et al.: Mg-chelatase I subunit 1 and $\mathrm{Mg}$-protoporphyrin IX methyltransferase affect the stomatal aperture in Arabidopsis thaliana. - J. Plant Res. 127: 553-563, 2014.

Tripathy B.C., Mohapatra A., Gupta I.: Impairment of the photosynthetic apparatus by oxidative stress induced by photosensitization reaction of protoporphyrin IX. - BBABioenergetics 1767: 860-868, 2007.

Tsang E.W., Yang J., Chang Q. et al.: Chlorophyll reduction in the seed of Brassica napus with a glutamate 1-semialdehyde aminotransferase antisense gene. - Plant Mol. Biol. 51: 191-201, 2003.

van Wijk K.J., Peltier J.B., Giacomelli L.: Isolation of chloroplast proteins from Arabidopsis thaliana for proteome analysis. Method. Mol. Biol. 355: 43-48, 2007.

Wang L., Yue C., Cao H. et al.: Biochemical and transcriptome analyses of a novel chlorophyll-deficient chlorina tea plant cultivar. - BMC Plant Biol. 14: 352, 2014.

Yu J., Zhang J., Zhao Q. et al.: Proteomic analysis reveals the leaf color regulation mechanism in chimera Hosta 'Gold Standard' leaves. - Int. J. Mol. Sci. 17: 346, 2016.

(C) The authors. This is an open access article distributed under the terms of the Creative Commons BY-NC-ND Licence. 